\title{
Diversity, dynamics and ecological analysis of flora of reclaimed soil
}

\author{
Kateryna Andrusevych ${ }^{1,2^{*}}$, Galina Zadorozhnaya ${ }^{1,3}$ \\ ${ }^{1}$ Dniprovsko-Orilskiy Nature Reserve, 52030 Obukhovka, Dniprovsk district, Dnipropetrovsk region, Ukraine \\ ${ }^{2}$ Department of Ecology and Technologies of Environmental Protection, National Technical University \\ "Dnipro Polytechnic", pr. Dmitry Yavornitskogo, 19, 49000, Dnipro, Dnipropetrovsk region, Ukraine \\ ${ }^{3}$ Department of Biochemistry and Physiology, Oles Honchar Dnipro National University, pr. Gagarina, 72, \\ 49010 Dnipro, Ukraine
}

\begin{abstract}
ANDrusevych, K., ZadorozhnAYA, G., 2019. Diversity, dynamics and ecological analysis of flora of reclaimed soil. Folia Oecologica, 46: 153-163.

The flora of vegetation cover of sod-lithogenic soil on loess loams was studied at a reclaimed site in the Nikopol manganese-ore basin. The control site is located on the black soil of the steppe area. The species composition of plants was studied in both sites annually for three years. Ecological analysis of the flora was carried out according to Raunkiaer's system of life-forms and Belgard's system of ecomorphs. It has been established that the floristic composition on the reclaimed site is significantly poorer than that of the steppe site. The reclaimed site was found to be have fewer species and a smaller number of families. The comparative inconstancy and dynamism of floristic composition on reclaimed soil is shown. The reclaimed ecosystem is distinguished by a significant share of the participation of annual and biennial plant forms. This indicates the anthropogenic transformation of the vegetation cover of the reclaimed soil. Also, a distinctive feature of the reclaimed soil flora is the smaller number of ecological groups of species. Such groups are united according to the ecological optimum to one of the environmental parameters.
\end{abstract}

\section{Key words}

Nikopol manganese-ore basin, phytoindication, reclamation, Ukraine

\section{Introduction}

The process of ecosystem destruction is an integral part of open pit mining (SHEORAN at al., 2010). When mineral resources are exploited in a quarry, the black soil of Ukraine is completely deprived of vegetation cover, wildlife and its humus horizon. Rocks are carried from different depths to the surface. They do not have a living phase and differ sharply in their properties from zonal soils (ZHUKOV and ZADOROZHNAYA, 2016). The settling of living organisms in the substrate begins upon contact with the atmosphere, soil formation processes occur, and the formation of ecosystems begins (ZHUKOV et al., 2012). Of great importance are questions of possible economic use of territories with reclaimed soils and their diagnostics (VORON, 2010). The study of the processes accompanying the reclamation provides the basis for a comprehensive assessment of the state of the environment (ANDRUSEVYCH and SHTIRTS, 2014).

Data on plant cover diversity are integral to a wide variety of soil quality indicators. Flora characteristics are used as an indicator of habitat environment assessment (Didukh, 2011, 2012; MatveEv, 2011). The activ-

*Corresponding author:

e-mail: eandrusevich.311089@gmail.com 
ity of organisms (both transformative (VERNADSKIY, 1978; LOVELOCK, 2000; KorZH, 2013a) and niche-forming (ODLING-SMEE et al., 2003)), indicates the mutual influence of organisms and their habitat (KoRzH, 2013a). The combination of conditions in which a species exists is the result of existing effects. This result is related to the concept of environmental capacity (KoRzH et al., 2016). Evaluation of the relative contribution of each factor in maintaining this capacity is extremely difficult (KoRzH et al., 2016). The habitat capacity can be used as an indicator of the "security" of a species. It can also be used as a limit to the implementation of its adaptive properties (KoRZH, 2013b). A complex generalized characteristic of environmental conditions can be obtained using the ecological characteristics of species of flora (DIDUH, 2011; ANDRUSEVICH and SHTIRTS 2014). The course of ecosystem and soil formation is generally reflected in the dynamic rearrangement of the plant community (ZADOROZHNA, 2018; ZADOROZHNA et al., 2018.). Studies on this theme have great prospects for use because they are practical and relatively simple to carry out.

The aim of this paper is assess of the dynamics of ecosystems on reclaimed soil by indicators of diversity, dynamics and data of ecological analysis of flora.

\section{Materials and methods}

The research was carried out at a reclaimed site of the Nikopol manganese ore basin for three years (2012-2014). The experimental site is located on sod-lithogenic soil on loess-like loams (YeterevsKa et al., 2008). According to IUSS Working Group WRB (2015) the soil is called Hyperskeletic Technosol (Calcaric, Supramollic, Vertic). Mining activity ceased 40 years ago, after which soil was added to make the site suitable for agriculture. At the time of the study, the land of the reclamation site had not used as an agricultural land for about 20 years.

The control site is located in a steppe area adjacent to the south-eastern slope of the Kamenistaya Gully (southern outskirts of Dnipro, Ukraine). On the north side of the steppe site there is a forest belt and an agricultural field. The soil of the control site is represented by black soil. According to IUSS Working Group WRB (2015) the soil is called Vermic Protovertic Chernozem (Katoloamic, Humic, Protocalcic). The control site is located on loess loam as it is the source rock for black soil. Meteorological data was taken at the meteorological stations of Nikopol and Dnipro (Table 1).

Species composition taken into consideration in the analysis of plant community. The names of species of plants and their systematic position is given according to the TARASOV (2012).

Ecological analysis of flora was carried out according to the classification of Raunkiaer's life-forms (RAUNKIÆR, 1937; Tarasov, 2012) and the ecological characteristic of species according to BELGARD (1971).

Plant life forms are represented by annual, biennial and perennial forms (ANDRUSEVICH and SHTIRTS, 2014). The share of perennial form in this paper was calculated as the sum of shares of perennial grasses, subshrubs, shrubs and trees.

Raunkiaer's life-forms (or climamorphs) are groups of plants separated by the position and method of protection of the regenerative buds during adverse periods (cold or dry). According to this classification, there are:

Class I: Phanerophytes $(\mathrm{Ph})$ - They are any tall plants visible all year round, which can afford to carry their perennial buds well above the ground, at least $25 \mathrm{~cm}$ up. This would include all trees, lianas, and virtually all shrubs in the structural classification.

Class II: Chamaeophytes (Ch) - They are low growing plants that are visible all the year-round, which carry their perennial buds up to $25 \mathrm{~cm}$ above the ground. They are more capable of handling rougher environments than phanerophytes because of their low stature (they are less exposed to the wind and receive some ground warming).

Class III: Hemicryptophytes (H) - They die back, to buds at ground surface during the unfavourable seasons. Their perennial buds lie close to the ground surface (above or below) and are often hidden by litter during unfavourable season, which protects them. So they are "half-hidden" plants.

Class IV: Cryptophytes (Cr) - The surviving buds or shoot apices in this group of plants are buried in the ground (or under water).

Class V: Therophytes (Th) - They get through adverse periods (seasonal or even years long) as seeds or spores. They go through their entire life cycle, from seed/ spore to seed/spore, within one growing season, which can be amazingly short.

Ecological forms according to BELGARD (1971) include:

1. Trophomorphs are groups of species united by

Table 1. Meteorological data of test sites

\begin{tabular}{cccccc}
\hline \multirow{2}{*}{ Year } & \multicolumn{2}{c}{ Reclamation site } & & \multicolumn{2}{c}{ Test site } \\
\cline { 2 - 3 } \cline { 5 - 6 } & $\begin{array}{c}\text { Precipitation } \\
(\mathrm{mm})\end{array}$ & $\begin{array}{c}\text { Average temperature } \\
\left({ }^{\circ} \mathrm{C}\right)\end{array}$ & & $\begin{array}{c}\text { Precipitation } \\
(\mathrm{mm})\end{array}$ & $\begin{array}{c}\text { Average temperature } \\
\left({ }^{\circ} \mathrm{C}\right)\end{array}$ \\
\hline 2012 & 312.9 & 18.3 & 322.70 & 17.6 \\
2013 & 205.4 & 17.3 & & 184.70 & 16.7 \\
2014 & 235.8 & 17.5 & 387.30 & 16.7 \\
\hline
\end{tabular}


their ecological optimum in relation to the trophicity (fertility) of the soil. Different trophomorphs prefer soils with different levels of mineralization of the soil solution. They are divided into oligotrophs (OgTr) (a group of plants that live on depleted soils), mesotrophs (MsTr) (a group of plants of medium-rich soils) megatrophs (MgTr) (plants of rich habitats).

2. Hygromorphs are groups of plant species that differ in the required moisture regime. Hygromorphs are divided into seven subgroups according to the degree of humidification: xerophytes (Ks) (plants of dry biotopes), mesoxerophytes (MsKs) (plants of dryish biotopes), xeromesophytes (KsMs) (plants of fresh, slightly moist biotopes), mesophytes (Ms) (plants of moist biotopes), hygromesophytes (HgMs) (plants of wet biotopes).

3. Heliomorphs are groups of species that need certain light conditions. They are divided into heliophytes (He) (an ecological group of plants whose ecological optimum corresponds to the light regime of open spaces), scioheliophytes $(\mathrm{ScHe})$ (the ecological optimum corresponds to the light regime of partly shaded forests with a latticed light structure), heliosciophytes (HeSc) (the ecological optimum corresponds to the light regime of semi-dense plantations of the penumbral structure).

4. Coenomorphs are ecological groups of plants that are tied to the environment of the community as a whole. They are divided into pratants (Pr) (meadow species), psammophytes (Ps) (plants of sandy habitats), ruderants (Ru) (weedy species), silvants (Sil) (forest species), stepants (St) (steppe species).

Methods of descriptive statistics are used in statistical calculations. As a measure of similarity used the Jaccard index, also known as Intersection over Union and the Jaccard similarity coefficient (Jaccard, 1901):

$$
\mathrm{K}_{\mathrm{J}}=\mathrm{c} /(\mathrm{a}+\mathrm{b}-\mathrm{c})
$$

where $\mathrm{a}$ is the number of species of the first test site, absent at the second test site, $\mathrm{b}$ is the number of species unique to the second test site, and $\mathrm{c}$ is the number of species common to both test sites.

\section{Results}

46 vascular plant species were identified in the vegetation cover of the reclaimed site, sod-lithogenic soil on loess loams, during the study period (Table 2). The composition of the flora of the experimental site changed significantly during the study period. So, 17 plant species were recorded in every year of the study period. They constitute $36.95 \%$ of the total number of registered species. The remaining components of the flora were not recorded every year. 15 species $(32.60 \%)$ were recorded for two years. 14 species $(30.43 \%)$ were registered in only one of the three years of research. The Jaccard index as a measure of similarity between the floristic composition of the test site in 2012 and 2014 amounted to 0.67 .
73 species of higher vascular plants were found in the flora of the steppe slope of the gully (Table 3 ). The composition of the flora of the control area also changed during the study period. On the black soil site, 49 plant species were identified in 2012, 63 species were identified in 2013, 60 species were identified in 2014. 42 plant species were recorded annually. This accounted for $57.53 \%$ of the total number of species. The remaining components of the flora were not recorded every year. 16 species $(21.91 \%)$ were present for 2 years. 15 species $(20.54 \%)$ were found once in three years of study. The Jaccard index between the data of the floristic composition of 2012 and 2014 at the control site is 0.75 . The Jaccard index averaged 0.28 when assessing the similarities between the species diversity of the reclamation and steppe sites.

An ecological analysis of the flora was carried out with the aim of making an integrated assessment of the conditions of the studied sites. Namely, moisture regimes, lighting, soil fertility conditions (trophicity) as a combination of existing environmental conditions.

The study found that approximately half of the plant species in the reclamation site were perennials. Their share in the list of flora varies from 45.45 to $55.17 \%$. The other half consisted of biennial plants (10.34-18.18\%) and annuals (33.33-36.36 \%) (Fig. 1).

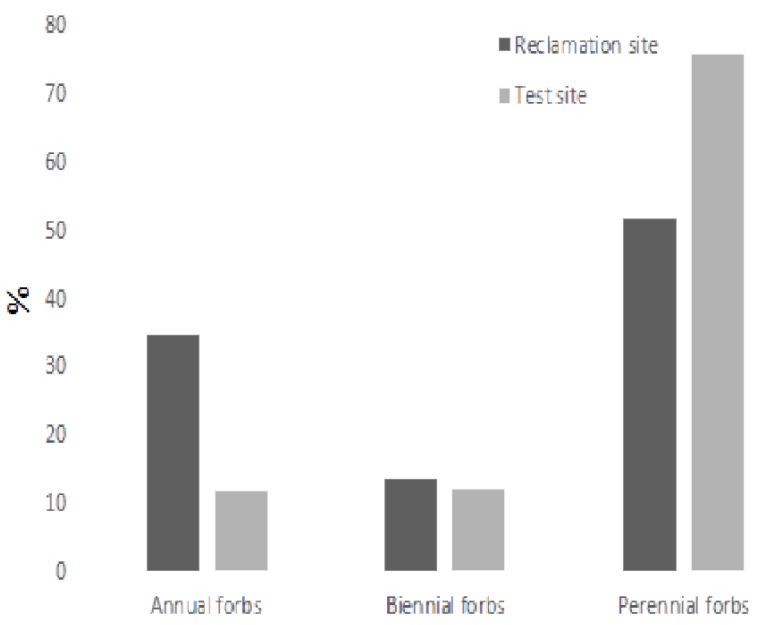

Fig. 1. The average values of the share of participation of plant life forms of the reclamation and the control sites.

The overwhelming majority of the flora species in the control area were representatives of perennial forms (71.43-81.63\%). The subordinate position was occupied by biennial (11.11-13.33\%) and annual plants (6.12$17.46 \%$ ) (Fig. 1).

The spectrum of Raunkiaer's life-forms of the plant species composition of the sod-lithogenic soils on loess loams was represented by 3 forms. Hemicryptophytes (with shares $(51.52-5.17 \%)$ dominated, therophytes (31.03-33.33\%) and cryptophytes (12.12-15.15\%) were in a subordinate position (Fig. 2). 
Table 2. List of plant species of the reclamation site for three years of research, their life forms and ectomorphs

\begin{tabular}{|c|c|c|c|c|c|c|c|c|c|c|}
\hline & Species of plants & \multicolumn{3}{|c|}{ Year } & $\begin{array}{l}\text { Life- } \\
\text { forms }\end{array}$ & $\begin{array}{l}\text { Raunkiaer's } \\
\text { life-forms }\end{array}$ & $\begin{array}{l}\text { Tropho- } \\
\text { morphs }\end{array}$ & $\begin{array}{l}\text { Hygro- } \\
\text { morphs }\end{array}$ & $\begin{array}{l}\text { Helio- } \\
\text { morphs }\end{array}$ & $\begin{array}{l}\text { Coeno- } \\
\text { morphs }\end{array}$ \\
\hline 1 & Achillea millefolium $\mathrm{L}$. & + & + & + & $\mathrm{PF}$ & $\mathrm{H}$ & $\operatorname{MgTr}$ & KsMs & $\mathrm{He}$ & $\operatorname{Pr}$ \\
\hline 2 & Aegilops cylindrica Host & - & - & + & $\mathrm{AF}$ & Th & OgMsTr & MsKs & $\mathrm{He}$ & Ptr \\
\hline 3 & $\begin{array}{l}\text { Agropyron pectinatum (M. } \\
\text { Bieb.) P. Beauv. }\end{array}$ & - & + & + & $\mathrm{PF}$ & $\mathrm{H}$ & $\operatorname{MsTr}$ & Ks & $\mathrm{He}$ & St \\
\hline 4 & Alyssum desertorum Stapf. & - & + & - & $\mathrm{AF}$ & Th & $\operatorname{MsTr}$ & MsKs & $\mathrm{He}$ & St \\
\hline 5 & Ambrosia artemisifolia $\mathrm{L}$. & + & - & + & $\mathrm{AF}$ & Th & $\mathrm{OgMsTr}$ & MsKs & $\mathrm{ScHe}$ & $\mathrm{Ru}$ \\
\hline 6 & $\begin{array}{c}\text { Anisantha tectorum (L.) } \\
\text { Nevski }\end{array}$ & + & + & + & $\mathrm{AF}$ & Th & $\operatorname{MgTr}$ & KsMs & $\mathrm{ScHe}$ & $\mathrm{St}$ \\
\hline 7 & Anthemis arvensis L. & - & + & - & $\mathrm{AF}$ & Th & $\operatorname{MsTr}$ & MsKs & $\mathrm{He}$ & St \\
\hline 8 & Artemisia absinthium $\mathrm{L}$. & + & + & + & $\mathrm{PF}$ & $\mathrm{H}$ & $\operatorname{Ms} \operatorname{Tr}$ & KsMs & $\mathrm{He}$ & St \\
\hline 9 & Bromus squrrosus L. & + & + & + & $\mathrm{AF}$ & Th & $\operatorname{MgTr}$ & MsKs & $\mathrm{ScHe}$ & St \\
\hline 10 & Centaurea diffusa Lam. & + & + & + & $\mathrm{BF}$ & $\mathrm{H}$ & $\operatorname{MsTr}$ & Ks & $\mathrm{He}$ & St \\
\hline 11 & Consolida regalis Gray & + & + & + & $\mathrm{AF}$ & Th & $\operatorname{MsTr}$ & MsKs & $\mathrm{ScHe}$ & $\mathrm{St}$ \\
\hline 12 & Convolvulus arvensis $\mathrm{L}$. & + & + & + & $\mathrm{PF}$ & $\mathrm{Cr}$ & $\operatorname{Ms} \operatorname{Tr}$ & MsKs & $\mathrm{ScHe}$ & St \\
\hline 13 & Crepis tectorum L. & + & - & - & $\mathrm{AF}$ & Th & $\operatorname{Ms} \operatorname{Tr}$ & MsKs & $\mathrm{He}$ & St \\
\hline 14 & Elytrigia repens (L.) Nevski & + & + & + & $\mathrm{PF}$ & $\mathrm{Cr}$ & $\operatorname{MsTr}$ & KsMs & $\mathrm{ScHe}$ & $\operatorname{Pr}$ \\
\hline 15 & Erigeron acris L. & - & - & + & $\mathrm{AF}$ & Th & $\operatorname{Ms} \operatorname{Tr}$ & MsKs & $\mathrm{He}$ & St \\
\hline 16 & Erysimum diffusum Ehrh. & - & + & + & $\mathrm{BF}$ & $\mathrm{H}$ & $\operatorname{MsTr}$ & Ks & $\mathrm{He}$ & St \\
\hline 17 & $\begin{array}{c}\text { Euphorbia stepposa Zoz. ex } \\
\text { Prokh. }\end{array}$ & - & + & - & PF & $\mathrm{H}$ & $\operatorname{MgTr}$ & Ks & $\mathrm{He}$ & St \\
\hline 18 & Falcaria vulgaris Bernh & + & - & + & PF & $\mathrm{H}$ & $\operatorname{MgTr}$ & Ks & $\mathrm{He}$ & St \\
\hline 19 & Festuca valesiaca Goud. s.l. & + & - & - & $\mathrm{PF}$ & $\mathrm{H}$ & $\operatorname{MgTr}$ & MsKs & $\mathrm{He}$ & St \\
\hline 20 & Lactuca serriola $\mathrm{L}$. & + & - & + & $\mathrm{AF}$ & Th & $\operatorname{MsTr}$ & $\mathrm{KsMs}$ & $\mathrm{He}$ & St \\
\hline 21 & $\begin{array}{c}\text { Lactuca tatarica }(\mathrm{L} .) \\
\text { C.A.Mey }\end{array}$ & - & + & + & PF & $\mathrm{Cr}$ & $\operatorname{MsTr}$ & $\mathrm{KsMs}$ & $\mathrm{He}$ & St \\
\hline 22 & Lepidium perfoliatum $\mathrm{L}$. & + & + & - & $\mathrm{AF}$ & Th & MsTr & MsKs & $\mathrm{He}$ & St \\
\hline 23 & Linaria genistifolia $\mathrm{L}$. & - & - & + & $\mathrm{PF}$ & $\mathrm{H}$ & Og-MsTr & Ks & $\mathrm{He}$ & St \\
\hline 24 & Lotus ucrainicus Klkov & + & + & - & $\mathrm{PF}$ & $\mathrm{H}$ & $\operatorname{MsTr}$ & KsMs & $\mathrm{He}$ & $\operatorname{Pr}$ \\
\hline 25 & Medicago lupulina L. & + & - & - & $\mathrm{AF}$ & $\mathrm{H}$ & $\operatorname{MsTr}$ & $\mathrm{KsMs}$ & $\mathrm{He}$ & $\operatorname{Pr}$ \\
\hline 26 & Medicago romanica Prod. & + & - & + & $\mathrm{PF}$ & $\mathrm{H}$ & $\operatorname{MgTr}$ & KsMs & $\mathrm{He}$ & $\operatorname{Pr}$ \\
\hline 27 & Medicago sativa $\mathrm{L}$. & + & + & + & $\mathrm{PF}$ & $\mathrm{H}$ & $\operatorname{Mg} \operatorname{Tr}$ & KsMs & $\mathrm{He}$ & $\operatorname{Pr}$ \\
\hline 28 & Melica transsilvanica Schur & + & + & + & $\mathrm{PF}$ & $\mathrm{H}$ & MsTr & KsMs & $\mathrm{ScHe}$ & $\mathrm{St}$ \\
\hline 29 & Melilotus albus Medik. & - & - & + & $\mathrm{BF}$ & $\mathrm{H}$ & $\mathrm{MsTr}$ & $\mathrm{HgMs}$ & $\mathrm{He}$ & $\operatorname{Pr}$ \\
\hline 30 & Melilotus officinalis (L.) Pall. & - & + & + & $\mathrm{BF}$ & $\mathrm{H}$ & $\operatorname{MsTr}$ & KsMs & $\mathrm{He}$ & $\operatorname{Pr}$ \\
\hline 31 & Onobrychis viciifolia Scop. & + & + & + & $\mathrm{PF}$ & $\mathrm{H}$ & $\mathrm{MsTr}$ & KsMs & $\mathrm{He}$ & $\mathrm{St}$ \\
\hline 32 & Poa pratensis $\mathrm{L}$. & - & + & & $\mathrm{PF}$ & $\mathrm{Cr}$ & $\operatorname{MsTr}$ & Ms & $\mathrm{He}$ & $\operatorname{Pr}$ \\
\hline 33 & Reseda lutea $\mathrm{L}$. & + & + & + & $\mathrm{AF}$ & Th & $\operatorname{MgTr}$ & KsMs & $\mathrm{He}$ & $\mathrm{St}$ \\
\hline 34 & Rosa canina $L$ & + & + & + & $\mathrm{AF}$ & Th & $\operatorname{MgTr}$ & KsMs & $\mathrm{He}$ & $\mathrm{St}$ \\
\hline 35 & Rumex confertus Willd. & - & - & + & $\mathrm{PF}$ & $\mathrm{H}$ & $\operatorname{MsTr}$ & KsMs & $\mathrm{ScHe}$ & St \\
\hline 36 & Securigera varia (L.) Lassen & + & + & + & $\mathrm{PF}$ & $\mathrm{Cr}$ & $\operatorname{MgTr}$ & KsMs & $\mathrm{ScHe}$ & $\operatorname{Pr}$ \\
\hline 37 & Senecio jacobaea L. & + & + & - & $\mathrm{PF}$ & $\mathrm{H}$ & $\operatorname{MsTr}$ & KsMs & $\mathrm{He}$ & $\operatorname{Pr}$ \\
\hline 38 & Seseli campestre Besser & + & + & + & $\mathrm{PF}$ & $\mathrm{H}$ & $\mathrm{MgTr}$ & MsKs & $\mathrm{He}$ & St \\
\hline 39 & Silene dichotoma Ehrh & & + & + & $\mathrm{AF}$ & Th & $\operatorname{MsTr}$ & KsMs & $\mathrm{ScHe}$ & St \\
\hline 40 & Sonchus arvensis L. & + & - & - & $\mathrm{PF}$ & $\mathrm{Cr}$ & $\operatorname{MgTr}$ & KsMs & $\mathrm{He}$ & St \\
\hline 41 & Stellaria holostea L. & - & + & - & $\mathrm{PF}$ & $\mathrm{H}$ & $\operatorname{MsTr}$ & $\mathrm{HgMs}$ & $\mathrm{HeSc}$ & Sil \\
\hline 42 & Taraxacum officinale Wigg. & + & + & - & $\mathrm{PF}$ & $\mathrm{H}$ & $\operatorname{MsTr}$ & KsMs & $\mathrm{ScHe}$ & St \\
\hline 43 & Tragopogon major Jacq. & + & + & + & $\mathrm{BF}$ & $\mathrm{H}$ & $\operatorname{MsTr}$ & MsKs & $\mathrm{ScHe}$ & St \\
\hline 44 & Vicia cracca $\mathrm{L}$. & + & + & + & $\mathrm{PF}$ & $\mathrm{H}$ & $\operatorname{MsTr}$ & $\mathrm{HgMs}$ & $\mathrm{He}$ & $\operatorname{Pr}$ \\
\hline 45 & Xanthium strumarium L. & - & + & + & $\mathrm{AF}$ & Th & $\operatorname{MsTr}$ & KsMs & $\mathrm{He}$ & St \\
\hline 46 & Xeranthemum anпиит L.. & - & + & + & $\mathrm{AF}$ & Th & $\operatorname{MsTr}$ & Ks & $\mathrm{He}$ & $\mathrm{St}$ \\
\hline & Total & 29 & 33 & 33 & & & & & & \\
\hline
\end{tabular}

“+”- identified; "-" - not identified; life-forms: AF - annual forbs, BF - biennial forbs, PF - perennial forbs; Raunkiaer's lifeforms: $\mathrm{Ph}$. - phanerophytes, $\mathrm{Ch}$. - chamaeophytes, $\mathrm{H}$ - hemicryptophytes, $\mathrm{Cr}$ - cryptophytes, $\mathrm{Th}$ - therophytes; trophomorphs: $\mathrm{OgTr}$ - oligotrophs, MsTr - mesotrophs, MgTr - megatrophs; hygromorphs: Ks - xerophytes, MsKs - mesoxerophytes, KsMs - xeromesophytes, Ms - mesophytes, HgMs - hygromesophytes; heliomorphs: He - heliophytes, ScHe - scioheliophytes, $\mathrm{HeSc}$ - heliosciophytes; coenomorphs: Pr - meadow species, Ps - plants of sandy habitats, Ru - weedy species, Sil - forest species, St - steppe species. 
Table 3. List of plant species of the steppe site for three years of research, their life forms and ecomorphs

\begin{tabular}{|c|c|c|c|c|c|c|c|c|c|c|}
\hline & \multirow{2}{*}{ Species of plants } & \multicolumn{3}{|c|}{ Year } & \multirow{2}{*}{$\begin{array}{l}\text { Life- } \\
\text { forms }\end{array}$} & \multirow{2}{*}{$\begin{array}{l}\text { Raunkiaer's } \\
\text { life-forms }\end{array}$} & \multirow{2}{*}{$\begin{array}{l}\text { Tropho- } \\
\text { morphs }\end{array}$} & \multirow{2}{*}{$\begin{array}{l}\text { Hygro- } \\
\text { morphs }\end{array}$} & \multirow{2}{*}{$\begin{array}{l}\text { Helio- } \\
\text { morphs }\end{array}$} & \multirow{2}{*}{$\begin{array}{l}\text { Coeno- } \\
\text { morphs }\end{array}$} \\
\hline & & 2012 & 2013 & 2014 & & & & & & \\
\hline 1 & Acer tataricum $\mathrm{L}$. & + & - & - & $\mathrm{PF}$ & $\mathrm{Ph}$ & MsTr & KsMs & $\mathrm{He}$ & Sil \\
\hline 2 & Achillea millefolium $\mathrm{L}$. & + & + & + & $\mathrm{PF}$ & $\mathrm{H}$ & $\operatorname{Mg} \operatorname{Tr}$ & KsMs & $\mathrm{He}$ & $\operatorname{Pr}$ \\
\hline 3 & Agrimonia eupatoria L. & + & + & - & $\mathrm{PF}$ & $\mathrm{H}$ & $\operatorname{MgTr}$ & KsMs & $\mathrm{ScHe}$ & St \\
\hline 4 & $\begin{array}{c}\text { Agropyron pectinatum (M. Bieb.) P. } \\
\text { Beauv. }\end{array}$ & - & + & - & $\mathrm{PF}$ & $\mathrm{H}$ & $\mathrm{MsTr}$ & $\mathrm{Ks}$ & $\mathrm{He}$ & St \\
\hline 5 & Ajuga genevensis L. & + & + & + & $\mathrm{PF}$ & $\mathrm{Cr}$ & $\mathrm{MsTr}$ & MsKs & $\mathrm{ScHe}$ & $\operatorname{Pr}$ \\
\hline 6 & Allium paniculatum $\mathrm{L}$. & - & - & + & $\mathrm{PF}$ & $\mathrm{Cr}$ & $\operatorname{MsTr}$ & MsKs & $\mathrm{He}$ & $\operatorname{Pr}$ \\
\hline 7 & Allium waldsteinii G. Don fil. & - & - & + & $\mathrm{PF}$ & $\mathrm{Cr}$ & $\operatorname{MsTr}$ & Ms & $\mathrm{He}$ & $\operatorname{Pr}$ \\
\hline 8 & Armeniaca vulgaris Lam. & + & + & + & $\mathrm{PF}$ & $\mathrm{Ph}$ & $\operatorname{MsTr}$ & MsKs & $\mathrm{He}$ & Sil \\
\hline 9 & Artemisia absinthium L. & & + & + & $\mathrm{PF}$ & $\mathrm{H}$ & $\operatorname{MsTr}$ & KsMs & $\mathrm{He}$ & $\mathrm{St}$ \\
\hline 10 & Artemisia austriaca Jacq. & + & + & + & $\mathrm{PF}$ & $\mathrm{H}$ & $\mathrm{MsTr}$ & Ks & $\mathrm{He}$ & St \\
\hline 11 & Asparagus officinalis $\mathrm{L}$. & + & + & + & $\mathrm{PF}$ & $\mathrm{H}$ & $\operatorname{Mg} \operatorname{Tr}$ & MsKs & $\mathrm{ScHe}$ & St \\
\hline 12 & Bromus squarrosus L. & - & + & + & $\mathrm{AF}$ & Th & $\operatorname{Mg} \operatorname{Tr}$ & MsKs & $\mathrm{ScHe}$ & St \\
\hline 13 & Calamagrostis epigeios (L.) Roth & - & + & + & $\mathrm{PF}$ & $\mathrm{Cr}$ & $\operatorname{Og} \operatorname{Tr}$ & Ms & $\mathrm{ScHe}$ & $\operatorname{Pr}$ \\
\hline 14 & Campanula patula $\mathrm{L}$ & + & + & + & $\mathrm{BF}$ & $\mathrm{H}$ & $\operatorname{MsTr}$ & Ms & $\mathrm{ScHe}$ & Sil \\
\hline 15 & Carduus acanthoides $L$. & + & + & + & $\mathrm{AF}$ & Th & $\operatorname{MsTr}$ & KsMs & $\mathrm{ScHe}$ & Sil \\
\hline 16 & Centaurea scabiosa L. & + & + & + & $\mathrm{PF}$ & $\mathrm{H}$ & $\operatorname{MgTr}$ & MsKs & $\mathrm{ScHe}$ & $\mathrm{St}$ \\
\hline 17 & $\begin{array}{c}\text { Chamaecytisus ruthenicus (Fisch. } \\
\text { Ex Wox.) Klasova }\end{array}$ & + & + & + & $\mathrm{PF}$ & $\mathrm{nPh}$ & $\mathrm{MsTr}$ & KsMs & $\mathrm{ScHe}$ & St \\
\hline 18 & Chondrilla juncea L. & + & + & + & $\mathrm{BF}$ & $\mathrm{H}$ & $\operatorname{Og} \operatorname{Tr}$ & MsKs & $\mathrm{He}$ & Ps \\
\hline 19 & Consolida regalis Gray & & + & + & $\mathrm{AF}$ & Th & $\operatorname{MsTr}$ & MsKs & $\mathrm{ScHe}$ & St \\
\hline 20 & Convolvulus arvensis $\mathrm{L}$. & + & + & + & $\mathrm{PF}$ & $\mathrm{Cr}$ & $\mathrm{MsTr}$ & MsKs & $\mathrm{ScHe}$ & $\mathrm{St}$ \\
\hline 21 & Crepis tectorum $\mathrm{L}$. & - & + & - & $\mathrm{AF}$ & $\mathrm{Th}$ & $\operatorname{MsTr}$ & MsKs & $\mathrm{He}$ & St \\
\hline 22 & Descurainia sophia L. & & + & - & $\mathrm{AF}$ & Th & $\operatorname{MsTr}$ & KsMs & $\mathrm{He}$ & $\mathrm{St}$ \\
\hline 23 & Dianthus fischeri Spreng. & - & - & + & $\mathrm{PF}$ & $\mathrm{H}$ & $\mathrm{Og} \operatorname{Tr}$ & MsKs & $\mathrm{ScHe}$ & Ps \\
\hline 24 & Elytrigia repens (L.) Nevski & + & + & + & $\mathrm{PF}$ & $\mathrm{Cr}$ & $\operatorname{MsTr}$ & KsMs & $\mathrm{ScHe}$ & $\operatorname{Pr}$ \\
\hline 25 & Eryngium campestre L. & + & + & + & $\mathrm{PF}$ & $\mathrm{Cr}$ & $\mathrm{MsTr}$ & Ks & $\mathrm{He}$ & St \\
\hline 26 & Erysimum diffusum Ehrh. & + & + & + & $\mathrm{BF}$ & $\mathrm{H}$ & $\operatorname{MsTr}$ & $\mathrm{Ks}$ & $\mathrm{He}$ & St \\
\hline 27 & Euphorbia stepposa Zoz. ex Prokh. & + & + & + & $\mathrm{PF}$ & $\mathrm{H}$ & $\operatorname{Mg} \operatorname{Tr}$ & Ks & $\mathrm{He}$ & $\mathrm{St}$ \\
\hline 28 & Euphorbia virgata Waldst. \& Kit & + & + & + & $\mathrm{PF}$ & $\mathrm{H}$ & $\operatorname{MsTr}$ & MsKs & $\mathrm{ScHe}$ & $\operatorname{Pr}$ \\
\hline 29 & Falcaria vulgaris Bernh. & + & + & + & $\mathrm{BF}$ & $\mathrm{H}$ & $\operatorname{Mg} \operatorname{Tr}$ & KsMs & $\mathrm{He}$ & $\mathrm{St}$ \\
\hline 30 & Festuca rubra L. & & + & + & $\mathrm{PF}$ & HK & $\operatorname{MgTr}$ & Ms & $\mathrm{ScHe}$ & $\operatorname{Pr}$ \\
\hline 31 & Festuca valesiaca Goud. s.l. & + & + & & $\mathrm{PF}$ & $\mathrm{H}$ & $\operatorname{Mg} \operatorname{Tr}$ & MsKs & $\mathrm{He}$ & St \\
\hline 32 & Galatella villosa (L.) Rchb.f. & + & + & + & $\mathrm{PF}$ & $\mathrm{Cr}$ & $\operatorname{MsTr}$ & Ks & $\mathrm{He}$ & St \\
\hline 33 & Galium hupanicum & + & - & + & $\mathrm{PF}$ & $\mathrm{H}$ & $\operatorname{MgTr}$ & MsKs & $\mathrm{He}$ & St \\
\hline 34 & Gleditsia triacanthos L. & + & + & + & $\mathrm{PF}$ & $\mathrm{Ph}$ & $\operatorname{MsTr}$ & MsKs & $\mathrm{He}$ & St \\
\hline 35 & Helichrysum arenarium (L.)Moench & + & + & + & $\mathrm{PF}$ & HK & OgMsTr & MsKs & $\mathrm{He}$ & St \\
\hline 36 & Herniaria polygama J. Gay & + & + & + & $\mathrm{AF}$ & Th & OgMsTr & MsKs & $\mathrm{ScHe}$ & St \\
\hline 37 & Hieracium umbellatum L. & & + & + & $\mathrm{PF}$ & $\mathrm{H}$ & OgMsTr & KsMs & $\mathrm{ScHe}$ & $\operatorname{Pr}$ \\
\hline 38 & Hieracium virosum & + & - & - & $\mathrm{PF}$ & $\mathrm{H}$ & $\operatorname{MsTr}$ & MsKs & $\mathrm{ScHe}$ & $\mathrm{St}$ \\
\hline 39 & $\begin{array}{l}\text { Hypericum elegans Stephan ex } \\
\text { Willd. }\end{array}$ & - & + & + & $\mathrm{PF}$ & $\mathrm{H}$ & $\mathrm{MsTr}$ & MsKs & $\mathrm{He}$ & St \\
\hline 40 & Koeleria cristata (L.) Pers. & + & + & + & $\mathrm{PF}$ & $\mathrm{H}$ & $\operatorname{MgTr}$ & Ks & $\mathrm{He}$ & St \\
\hline 41 & Lactuca serriola $\mathrm{L}$. & & + & + & $\mathrm{AF}$ & Th & $\operatorname{MsTr}$ & KsMs & $\mathrm{He}$ & St \\
\hline 42 & Lepidium perfoliatum L. & - & + & - & $\mathrm{AF}$ & Th & $\operatorname{MsTr}$ & MsKs & $\mathrm{He}$ & $\mathrm{St}$ \\
\hline 43 & $\begin{array}{l}\text { Limonium sareptanum (A. Becker) } \\
\text { Gams }\end{array}$ & + & + & + & $\mathrm{PF}$ & $\mathrm{H}$ & $\operatorname{MgTr}$ & Ks & $\mathrm{He}$ & St \\
\hline 44 & Linaria genistifolia L. & - & + & + & $\mathrm{PF}$ & $\mathrm{H}$ & $\begin{array}{l}\text { Og- } \\
\text { MsTr }\end{array}$ & Ks & $\mathrm{He}$ & St \\
\hline 45 & Medicago romanica Prod. & + & + & + & $\mathrm{PF}$ & $\mathrm{H}$ & $\mathrm{MgTr}$ & KsMs & $\mathrm{He}$ & $\operatorname{Pr}$ \\
\hline 46 & Medicago sativa (L.) Mill. & + & + & + & $\mathrm{PF}$ & $\mathrm{H}$ & $\operatorname{MgTr}$ & KsMs & $\mathrm{He}$ & $\operatorname{Pr}$ \\
\hline 47 & Melica transsilvanica Schur & - & + & + & $\mathrm{PF}$ & $\mathrm{H}$ & $\operatorname{MsTr}$ & KsMs & $\mathrm{ScHe}$ & St \\
\hline 48 & Melilotus officinalis (L.) Pall. & - & + & + & $\mathrm{BF}$ & $\mathrm{H}$ & $\operatorname{MsTr}$ & KsMs & $\mathrm{He}$ & $\operatorname{Pr}$ \\
\hline 49 & Morus alba $\mathrm{L}$. & + & + & + & $\mathrm{PF}$ & $\mathrm{Ph}$ & $\mathrm{MsTr}$ & MsKs & $\mathrm{ScHe}$ & Sil \\
\hline 50 & Nonea pulla DC. & + & + & + & $\mathrm{PF}$ & $\mathrm{H}$ & $\mathrm{MsTr}$ & MsKs & $\mathrm{He}$ & $\mathrm{St}$ \\
\hline 51 & Otites wolgensis (Hornem.) Grossh. & + & + & + & $\mathrm{BF}$ & $\mathrm{H}$ & $\operatorname{MgTr}$ & MsKs & $\mathrm{He}$ & St \\
\hline 52 & Phalacroloma annuиm (L.) Dumort & - & - & + & $\mathrm{AF}$ & Th & $\operatorname{MsTr}$ & MsKs & $\mathrm{ScHe}$ & $\mathrm{Ru}$ \\
\hline
\end{tabular}


Table3. List of plant species of the steppe site for three years of research, their life forms and ecomorphs - continued.

\begin{tabular}{|c|c|c|c|c|c|c|c|c|c|c|}
\hline & \multirow{2}{*}{ Species of plants } & \multicolumn{3}{|c|}{ Year } & \multirow{2}{*}{$\begin{array}{l}\text { Life- } \\
\text { forms }\end{array}$} & \multirow{2}{*}{$\begin{array}{l}\text { Raunkiaer's } \\
\text { life-forms }\end{array}$} & \multirow{2}{*}{$\begin{array}{l}\text { Tropho- } \\
\text { morphs }\end{array}$} & \multirow{2}{*}{$\begin{array}{l}\text { Hygro- } \\
\text { morphs }\end{array}$} & \multirow{2}{*}{$\begin{array}{l}\text { Helio- } \\
\text { morphs }\end{array}$} & \multirow{2}{*}{$\begin{array}{l}\text { Coeno- } \\
\text { morphs }\end{array}$} \\
\hline & & 2012 & 2013 & 2014 & & & & & & \\
\hline 53 & Poa pratensis $\mathrm{L}$. & + & + & + & $\mathrm{PF}$ & $\mathrm{Cr}$ & $\operatorname{MsTr}$ & Ms & $\mathrm{He}$ & $\operatorname{Pr}$ \\
\hline 54 & Potentilla argentea $\mathrm{L}$. & + & + & + & $\mathrm{PF}$ & $\mathrm{H}$ & OgMsTr & KsMs & $\mathrm{ScHe}$ & $\operatorname{Pr}$ \\
\hline 55 & Potentilla erecta L. & - & + & + & $\mathrm{PF}$ & $\mathrm{Cr}$ & $\mathrm{MsTr}$ & $\mathrm{HgMs}$ & $\mathrm{ScHe}$ & $\operatorname{Pr}$ \\
\hline 56 & Potentilla obscura Willd. & + & - & & $\mathrm{PF}$ & $\mathrm{H}$ & $\operatorname{MgTr}$ & KsMs & $\mathrm{He}$ & $\operatorname{Pr}$ \\
\hline 57 & Reseda lutea $\mathrm{L}$. & + & + & + & $\mathrm{AF}$ & Th & $\operatorname{MgTr}$ & KsMs & $\mathrm{He}$ & St \\
\hline 58 & $\begin{array}{c}\text { Salvia nemorosa L. (Klokov et } \\
\text { Pobed.) Soó }\end{array}$ & + & + & + & $\mathrm{PF}$ & $\mathrm{H}$ & $\mathrm{MsTr}$ & Ms & $\mathrm{ScHe}$ & Sil \\
\hline 59 & Securigera varia (L.) Lassen) & + & + & + & $\mathrm{PF}$ & $\mathrm{Cr}$ & $\operatorname{Mg} \operatorname{Tr}$ & KsMs & $\mathrm{ScHe}$ & $\operatorname{Pr}$ \\
\hline 60 & Senecio jacobaea L. & + & + & + & $\mathrm{PF}$ & $\mathrm{H}$ & $\operatorname{MsTr}$ & KsMs & $\mathrm{He}$ & $\operatorname{Pr}$ \\
\hline 61 & Senecio vernalis Waldst. Et Kit & - & + & - & $\mathrm{AF}$ & $\mathrm{H}$ & $\begin{array}{l}\text { Og- } \\
\text { MsTr }\end{array}$ & KsMs & $\mathrm{ScHe}$ & $\operatorname{Pr}$ \\
\hline 62 & Seseli campestre Besser & + & + & + & $\mathrm{PF}$ & $\mathrm{H}$ & $\operatorname{MgTr}$ & MsKs & $\mathrm{He}$ & St \\
\hline 63 & Silene dichotoma Ehrh. & & + & & $\mathrm{AF}$ & Th & $\mathrm{MsTr}$ & KsMs & $\mathrm{ScHe}$ & St \\
\hline 64 & Stachys transsilvanica Schur & + & + & + & $\mathrm{PF}$ & $\mathrm{H}$ & OgMsTr & MsKs & $\mathrm{He}$ & St \\
\hline 65 & Stellaria holostea $\mathrm{L}$. & & + & & $\mathrm{PF}$ & $\mathrm{H}$ & $\mathrm{MsTr}$ & $\mathrm{HgMs}$ & $\mathrm{HeSc}$ & Sil \\
\hline 66 & Stipa capillata $\mathrm{L}$. & + & + & + & $\mathrm{PF}$ & $\mathrm{H}$ & $\begin{array}{l}\text { MgMsT } \\
\mathrm{r}\end{array}$ & Ks & $\mathrm{He}$ & St \\
\hline 67 & Teucrium polium $\mathrm{L}$. & + & + & + & $\mathrm{PF}$ & $\mathrm{Ch}$ & $\operatorname{MsTr}$ & Ks & $\mathrm{He}$ & St \\
\hline 68 & Thymus marschallianus Willd. & + & + & + & $\mathrm{PF}$ & $\mathrm{Ch}$ & MsTr & Ks & $\mathrm{He}$ & St \\
\hline 69 & Tragopogon major Jacq. & + & + & + & $\mathrm{BF}$ & $\mathrm{H}$ & $\mathrm{MsTr}$ & MsKs & $\mathrm{ScHe}$ & $\mathrm{St}$ \\
\hline 70 & Verbascum phoeniceum L. & + & + & & $\mathrm{PF}$ & $\mathrm{H}$ & $\mathrm{MsTr}$ & MsKs & $\mathrm{ScHe}$ & St \\
\hline 71 & Verbascum thapsus L. & & & + & $\mathrm{BF}$ & $\mathrm{H}$ & $\operatorname{Og} \operatorname{Tr}$ & KsMs & $\mathrm{ScHe}$ & $\operatorname{Pr}$ \\
\hline 72 & Veronica steppacea Kotov & + & + & + & $\mathrm{PF}$ & $\mathrm{H}$ & $\operatorname{MsTr}$ & Ks & $\mathrm{He}$ & St \\
\hline \multirow[t]{2}{*}{73} & Viola ambigua Waldst. \& Kit. & + & - & + & $\mathrm{PF}$ & $\mathrm{H}$ & $\operatorname{Mg} \operatorname{Tr}$ & MsKs & $\mathrm{ScHe}$ & St \\
\hline & Total & 49 & 63 & 60 & & & & & & \\
\hline
\end{tabular}

For key to abbreviations see Table 2.

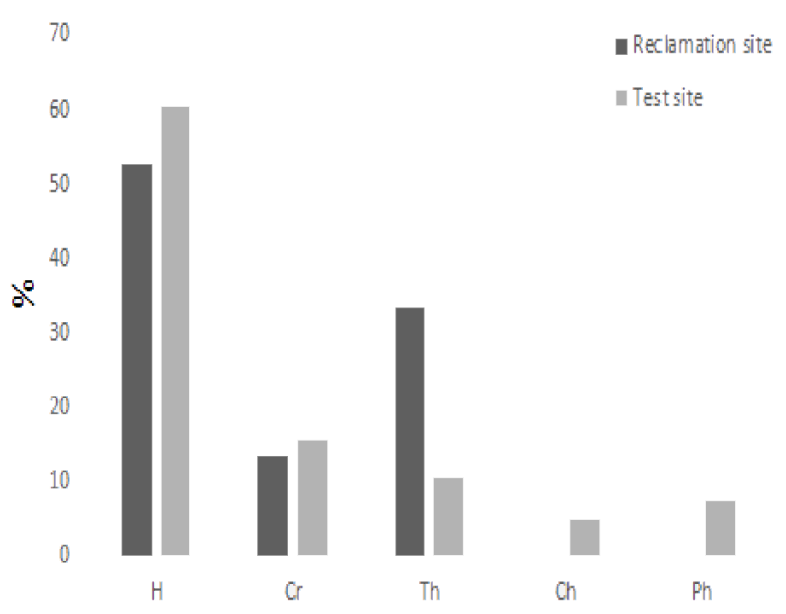

Fig. 2. The average values of the share of participation of Raunkiaer's life-forms of the reclamation and the control sites. $\mathrm{H}$ is hemicryptophytes, $\mathrm{Cr}$ is cryptophytes, $\mathrm{Th}$ is therophytes, $\mathrm{Ch}$ is chamaeophytes, $\mathrm{Ph}$ is phanerophytes

Raunkiaer's life-forms of the plant species composition of the black soil site were represented by 5 forms. Hemicryptophytes occupied a dominant position (58.33-63.27\%). Cryptophytes (14.29-18.33\%), therophytes (6.12-14.30\%), phanerophytes $(6.35-10.20 \%)$ and chamaeophytes $(4.76-6.12 \%)$ were in the subordinate position (Fig. 2).
The trophomorphs of the plant species composition of the reclaimed soil were represented by megatrophs (a group of species that prefer soils with a high concentration of soil solution) and mesotrophs (a group of species that prefer soils with a moderate concentration of soil solution). The share of mesotrophs was somewhat higher (58.62-72.73\%) than megatrophs (27.27-41.38\%). Trophmorphs of the control site were represented by mesotrophs (57.14-68.25\%), megatrophs (26.98-40.82\%) and oligotrophs (2.04-6.67\%) (Fig. 3).

In the spectrum of hygromorphs, the species composition of plants in the experimental area was dominated by xeromesophytes $(51.52-58.62 \%)$ and mesoxerophytes (24.24-34.48\%). The share of xerophytes was slightly less (3.45-15.15\%). Hygromesophytes were recorded with a participation share of $3.45-5.19 \%$ (Fig. 4).

Hygromorphs in the species composition of plants on the chernozem were very diverse: mesoxerophytes (36.51-42.86\%), xeromesophytes $(28.33-31.75 \%)$, xerophytes (20.00-22.45\%), mesophytes (6.12-10.00\%) and hygromesophytes (1.67-3.17\%). In 2012 hygromesophytes were not recorded (Fig. 4).

Among the heliomorphs, three forms were found on the both test sites. Heliophytes were also dominant at the reclamation site $(65.52-66.67 \%)$ and in the control site (57.14-61.22\%). Scioheliophytes occupied a subdominant position. Their representation was on average $9 \%$ 


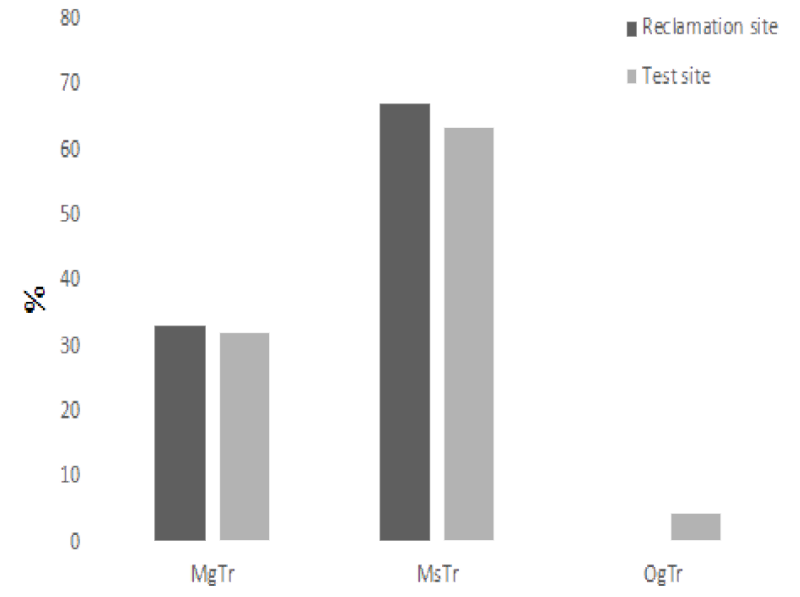

Fig. 3. The average values of the share of participation of trophomorphs of the reclamation and the control sites. MgTr is megatrophs; $\mathrm{MsTr}$ is mesotrophs; $\mathrm{Og} \mathrm{Tr}$ is oligotrophs.

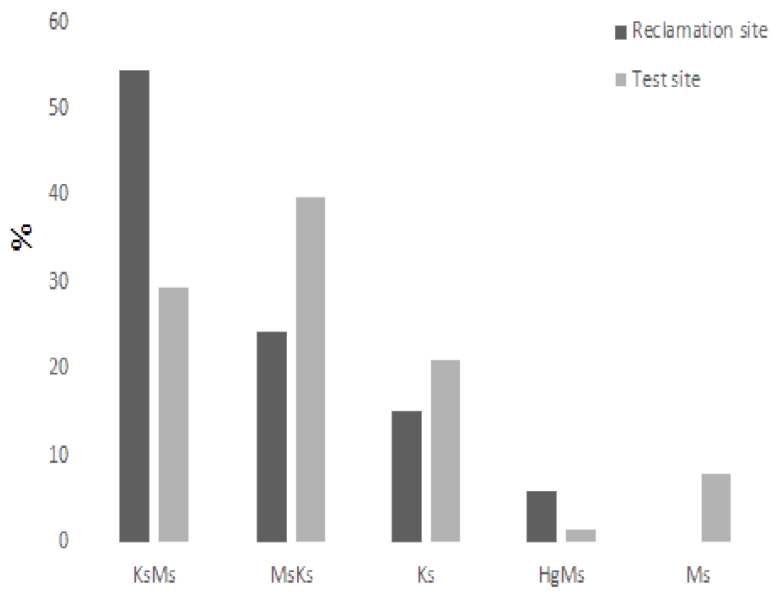

Fig. 4. The average values of the share of participation of hygromorphs of the reclamation and the control sites. $\mathrm{KsMs}$ is xeromesophytes; MsKs is mesoxerophytes; Ks is xerophytes; $\mathrm{HgMs}$ is hygromesophytes; Ms is mesophytes.

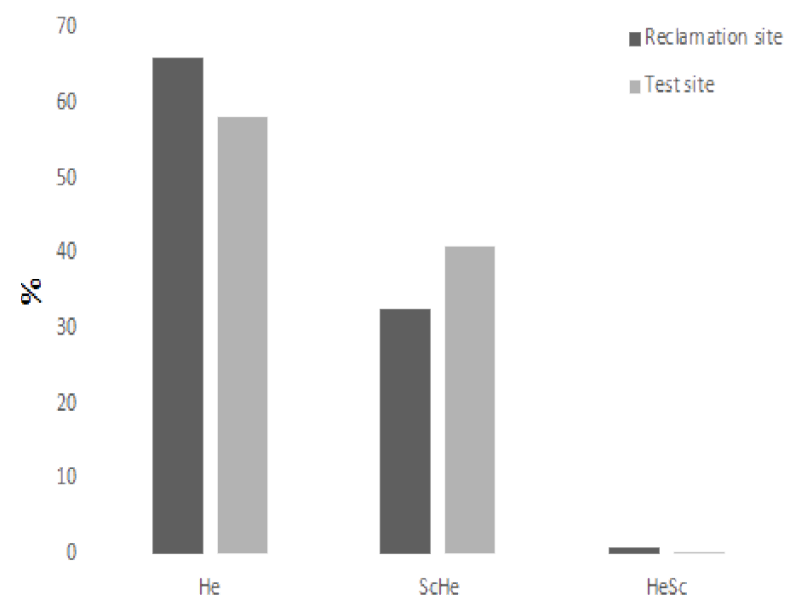

Fig. 5. The average values of the share of participation of heliomorphs of the reclamation and the control sites.

$\mathrm{He}$ is heliophytes; $\mathrm{ScHe}$ is scioheliophytes; $\mathrm{HeSc}$ is heliosciophytes.

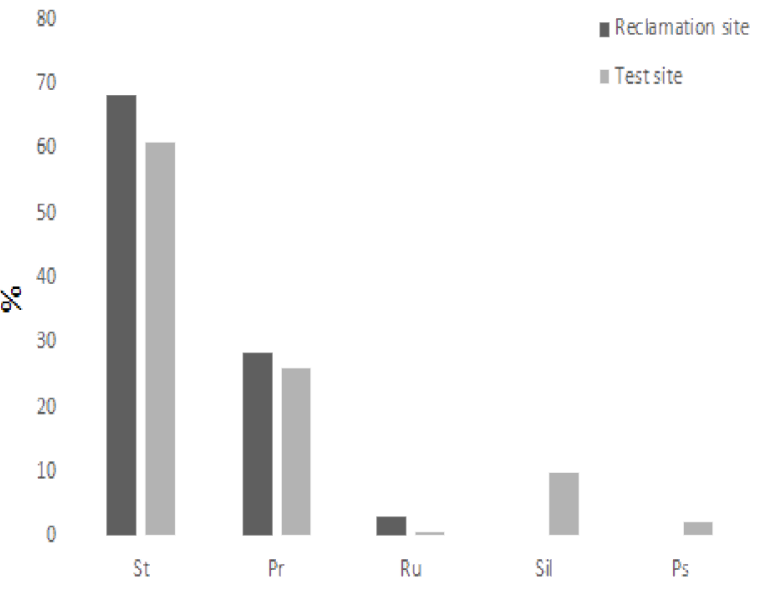

Fig. 6. The average values of the share of participation of coenomorphs of the reclamation and the control sites. St is steppe species; Pr is meadow species; Ru is weedy species;

Sil is forest species; Ps is plants of sandy habitats.

higher at the control steppe site. In 2013, one plant species belonging to heliosciophytes was recorded at both sites (Fig. 5).

Steppe species had the highest share at both sites (65.52-69.70\% - on the reclamation site, 56.67-63.49\% on the control site), but on the reclaimed soil the share was several percent higher. The remaining plant species of the experimental site were meadow species (27.27-31.03\%). One weed was recorded in this site during the study period. In addition to steppe species, meadow (22.45-30.00\%) and forest species $(8.33-12.24 \%)$ were observed in the flora of the control steppe site. One weed plant and two psammophytes species (plant of sandy habitats) were recorded in the control area during the study period (Fig. 6).

The presence of the invasive and quarantine species Ambrosia artemisifolia in both areas should be noted. Also, invasive species Phalacroloma annuum and Gleditsia triacanthos were found in the control site, and species Centaurea diffusa and Xanthium strumarium were found in the reclaimed site. Invasive species are listed according to ZaVIALOVA (2017), quarantine according to the Instructions for the identification, localization and elimination of source of quarantine weeds, approved by order of the Ministry of Agrarian Policy of Ukraine (27.01.2005 N 40).

\section{Discussion}

The results of the study showed that the flora of the reclamation site was significantly poorer than the flora on the black soil in the virgin steppe. The number of plant species in the control area was 1.48 times greater than in the experimental one. The excess of the number of steppe flora families was even more significant, by 1.69 times. This situation was observed throughout the three years of research. At the same time, the share of the dominant plant species at the reclamation site was almost twice the 
share at the steppe site. This suggests comparative poverty and homogeneity of the vegetation cover of the reclamation soil. Almost half of the species of virgin steppe flora (48.05\%) were recorded annually. This figure does not reach even one third of the whole list of plants on the reclamation soil $(28.84 \%)$. This indicates the comparatively inconstant and dynamic floristic composition of an artificial ecosystem. This is confirmed by the calculation of the measure of similarity. The value of the Jaccard index of flora diversity of the reclamation site is significantly lower than the values of the Jacquard index of flora of the control site ( 0.75 and 0.68 , respectively).

A characteristic feature of the flora of the both studied ecosystems is the dominance in the species composition of a small number of families. This phenomenon is characteristic of both artificial and natural ecosystems. According to TOKHTAR (2013), this indicates the presence of anthropogenic influence on both sites. However, the lifetime of the studied ecosystems varies significantly. When creating a site for reclamation, such components of the ecosystem as the relief and natural soil cover are completely transformed, the vegetation cover is formed a new on a new substrate. The control steppe site on the slope of a steppe gully is also subject to anthropogenic impact. This is expressed in sporadic weeding out of vegetation due to grazing. It is also likely to be subject to washout of pesticides from adjacent agricultural land, which is located higher than the control site. The share of the permanent components of the flora at the experimental and control sites differ by more than 1.6 times. This indicates different degrees of anthropogenic influences. Accordingly, the findings suggest the more unstable state of the artificial ecosystem flora, which confirms our previous conclusions.

The life forms of plants as a complex of habitual features emerging in the course of adaptive evolution reflect the complex relationship between plant species and environmental conditions in an integral form (SEMENOVATyan-ShansKaya, 1954; Sluginova, 2009). As is well known, annual and biennial species are practically absent in stable formed plant communities (IPATOV et al., 1996). An increase in their number is usually associated with the anthropogenic transformation of the vegetation cover (ILYINA, 2003). The prevalence of perennial plants in the community in the spectrum of flora life forms of the control site indicates a high degree of formation and stability of the community. Analysis of the ratio of flora life forms at the reclamation site showed a sufficiently large proportion of perennial plants. This indicates the process of naturalization of the plant community. However, the larger percentage of annual and biennial plant forms $(x=23.98 \%)$ at the experimental site compared to the control site indicates the relatively young age of the ecosystem. The experimental site was founded in 1970-1971. Until the beginning of the 2000s, it was cultivated as agricultural land.

Climamorphs or Raunkiaer's life-forms are formed historically as a result of the adaptation of plants to the climatic conditions of the environment (RAUNKIÆR, 1937; Stepanovskih, 2001; Andrusevich and Shtirts, 2014).
They characterize the adaptability of various plant species to the effect of unfavourable climatic and weather conditions during the winter period (MatveEv, 2011). Such spectra are used to analyze the vegetation of different communities of the same climatic zone (BELGARD, 1971). It is considered by some that climamorph types are too extensive and heterogeneous to serve as climate indicators (STEPANOVSKIH, 2001). We present the flora data of two test sites of the same climatic zone as a relative indicator of the ecological capacity of biotopes. According to Abduloeva and Solomakhi (2011), the more complex the organization of the community and the higher the ecological capacity of the habitat, the more diverse is the spectrum of the climamorph (life forms according to Raunkiaer). According to our data, the representation of climamorph types of reclaimed soil is somewhat less than in the virgin steppe site. The limiting factor may be the variability and a certain degree of extremes of the ecological regimes of the environment. Extremity is due to the low content of organic matter in reclaimed soil, severe shrinkage, wide variations in the temperature of the anthropogenic soil, and a high content of lumpy fractions in the aggregate composition (ANDRUSEVICH and LAGUNINA, 2013; ANDRUSEVICH and LYADSKA 2014; ZHUKOV and ZADOROZHNAYA, 2015, 2017).

The ecomorphic analysis proposed by BELGARD (1971), is an apparatus for studying ecosystems, built on the use of information about the requirements of the ecosystem populations for environmental parameters (temperature, light, nutrients, humidity) (CHERNYSHENKo and LysenKo, 2008; ZhuKov, 2009). All types of plant communities are coordinated by a series of gradients spatial, temporal, chemical, physical, etc. The variety of ecological forms allows different species to coexist and effectively distribute among themselves resources in time and space according to their needs (ABDULOEVA and SLOMAKHA, 2011). A distinctive feature of the reclaimed soil flora is the smaller number of ecomorphs - groups of species combined by ecological optimum to one of the environmental parameters.

According to the results of an ecomorphic (ecological) analysis of the spectrum of the trophophs of both polygons, mesotrophs dominate, which indicates that the soils of the studied sites are medium rich (medium fertile). However, the representation of megatrophs is also significant $-32.99 \%$ and $32.04 \%$ for reclaimed soil and black soil, respectively. This indicates a local presence of places with a higher content of mineral substances in the soil solution. The number of the trophomorphs at the control site is larger; it includes oligotrophic species $($ mean $=4.49 \%$ ).

In the spectrum of the hygromorphs of the flora of the test site, xeromesophytes, that is, plants of fresh biotopes, prevail. In the control site mesoxerophytes dominated - plants of rather dry habitats. However, the number of ecological groups of plants that require different moisture regimes was greater on the black soil in two out of the three years of research. 
The heliomorph spectra of both the studied sites are identical. They are dominated by heliophytes, an ecological group of plants, the ecological optimum of which corresponds to the light regime of open spaces.

The number of environmental groups associated with different habitats was also smaller at the experimental site. Steppe, meadow and weed species were recorded on the reclaimed soil site. The flora of the control site also included forest and sandy habitats.

It can be argued the ecological capacity of the environment of an artificial ecosystem is substantially less. The possibility of the existence of a certain number of organisms in the existing conditions of the reclamation site is significantly lower. The relatively low capacity of the medium exerts a kind of pressure on the corresponding group of organisms. This pressure can in a certain way limit various vital manifestations - distribution, number, reproduction, etc. (KoRzH et al, 2016; KIRILOV and BANOv, 2017; ButA et al., 2019).).

As mentioned earlier, the formation of the structure of the plant group is directly dependent on the influence of the environment (Montagu et al., 2001; Vanags et al., 2004; Godefroid and Koedam, 2003). Changes of the soil properties as a component of the environment for plants are of paramount importance (CHESSON, 2000). The intraspecific and interspecific relationships of plants of the virgin steppe have long been formed. The transformation of the environment here is relatively slow, the habitat capacity is higher. In anthropogenic soil, soil formation processes proceed at an increased rate (GERASIMOVA et al., 2003; ZADOROZHNA, 2018). This is confirmed by accelerated changes in the species composition of the plant cover. At the same time, anthropogenic soil is considered as a simpler system with low buffering capacity (GHOSE, 2001; AnAND et al., 2002; SERAFIM et al, 2008). The comparative poverty of the composition and ecological forms of the flora is explained by the small variety of ecological conditions of the artificial ecosystem, the relatively low level of its environmental capacity (ULLAH and WOLKENHAUER, 2011). The reason for this is the comparative youth of the anthropogenic ecosystem. About 42-45 years have passed from the beginning of the technical reclamation stage. The rapid change in species composition over this time indicates the variability of environmental regimes for vegetation of anthropogenic soil.

\section{Conclusions}

During the three years of research, it was found that the number of plant species at the reclamation site was 1.48 times less than at the steppe site. Changes in the flora composition of the reclamation site proceeded at a faster rate: out of the total number of plant species, $28.84 \%$ were recorded annually. This indicator in the control area was almost twice as high (48.05\%).

According to the floral ecological analysis of the life forms system, a greater number of annual and biennial plant forms grow on the reclamation site. This indicates the presence of anthropogenic load.

Analysis of the ratio of flora life forms at the reclamation site showed a sufficiently large proportion of perennial plants. This indicates the process of naturalization of the plant community. However, the larger percentage of annual and biennial plant forms $(23.98 \%)$ at the experimental site compared to the control site indicates the relatively young age of the ecosystem.

A distinctive feature of the reclamation site flora is the smaller number of ecological groups of species. These groups are united according to the ecological optimum to one of the environmental parameters. The flora of the reclamation site demonstrates a smaller variety of ecological plant forms according to position and way of protecting restoration buds during unfavourable periods, according to the ecological optimum in relation to soil fertility, moisture regime, lighting conditions, and also in relation to the plant community environment as a whole.

\section{References}

Abduloeva, O.S., Solomaha, V.A., 2011. Fitotsenologiya [Phytocenology]. Kyiv: Vydavatel'stvo Ukrainskoho Fitosotsiolohichnoho Tsentru. 450 p.

Anand, M., Tucker, B.C., Desrochers, R., 2002. Ecological monitoring of terrestrial ecosystem recovery from manmade perturbation: assessing community complexity. In Proceedings of the 10th international conference on modelling, monitoring and management of air pollution. Segovia, Spain, July 1-3, 2002. Southampton: WIT Press, p. 341-350.

ANDrusevich, E.V., Lagunina, V.G., 2013. Harakteristika usadki dernovo-litogennyih pochv na lessah po profilyu [Shrinkage characteristic sod-lithogenic soils on loess in the profile]. In Proceedings of the international scientific conference "From conservation to sustainable use of nature" Donetsk, 20-22 March 2013. Donetsk, p. 101-103.

Andrusevich, K.V., LyadsKa, I.V., 2014. Dinamika fizichnyih vlastivostey dernovo-litogennih gruntiv na chervono-buryih glyinah za profilem [Dynamics of the physical properties of sod-lithogenic soil on the red-brown clays the profile]. Visnyk of Lviv University. Biological Series, 18: 75-79.

Andrusevich, E.V., ShtiRts, Yu.A., 2014. Ekologicheskoe raznoobrazie rastitelnogo pokrova tehnozemov uchastka rekultivatsii Nikopolskogo margantsevorudnogo basseyna [Ecological diversity of vegetation on lithogenic soil in the reclamation land of the Nicopol manganese ore basin]. Industrial Botany, 14: 115-127.

Belgard, A.L., 1971. Stepnoe lesovedenie [Steppe forestry]. Moscow: Lesnaya promyshlennost. 336.

Buta, M., Blaga, G., Paulette, L., Păcurar, I., Roşca S., Borsai, O., Grecu, F., Sînziana, P., Negruşier, C., 2019. Soil reclamation of abandoned mine lands by revegetation in northwestern part of Transylvania: a 40-year retrospective study. Sustainability, 11 (12): 3393. https://doi. org/10.3390/su11123393 
Chernyshenko, V.S., Lysenko, J.J., 2008. Jekomorficheskii analiz A.L. Bel'garda kak teoreticheskaya osnova dlya matematicheskogo prognozirovaniya dinamiki populyacii [Belgard's ecomorph analysis as a theoretical basis for the mathematical prediction of population dynamics]. Ecology and Noospherology, 19 (1-2): 19-30.

Chesson, P., 2000. Mechanisms of maintenance of species diversity. Annual Review of Ecology, Evolution, and Systematics, 31: 343-366.

DidukH, YA.P., 2011. The ecological scales for the species of Ukrainian flora and their use in synphytoindication. Kyiv: Vydavatel'stvo Ukrainskoho Fitosotsiolohichnoho Tsentru. 176.

DiDUKH, YA.P., 2012. Osnovy bioindykatsii [Fundamentals of bioindication]. Kyiv: Naukova dumka. 344 p.

Gerasimova, M.I., Stroganova, M.N., Mozharova, M.V., Prokofieva T.V., 2003. Antropogennye pochvy (genezis, geografiya, rekul 'tivaciya) [Anthropogenic soils (genesis, geography, recultivation]. Smolensk: Oykumena. 270 p.

Ghose, M. K., 2001. Management of topsoil for geo-environmental reclamation of coal mining areas. Environmental Geology, 40: (11): 1405-1410. https://doi.org/10.1007/ s002540100321

Godefroid, S., Koedam, N., 2003. How important are large vs. small forest remnants for the conservation of the woodland flora in an urban context? Global Ecology and Biogeography, 12: 287-298. https://doi.org/10.1046/ j.1466-822X.2003.00035.X

ILYINA, V.N., 2003. Vlijanie stepnyh pozharov na sostojanie cenopopuljacij kopeechnikov Samarskogo regiona [Influence of wildfires on state of cenopopulation of Hedýsarum in Samara region]. In Perspektiva-2003. Materialy Vserossiiskoi nauchnoi konferentsii studentov, aspirantov i molodykh uchenykh. 4. Nalchik: Kabardino-Balkarskij Gosudarstvennyj Universitet, p. 28-31.

Instrukcyja z vyjavljennja, lokalizaciiita likvidacii vognyshh karantynny bur'janiv (Zatverdzheno Nakaz Ministerstva agrarnoj polityky Ukrainy 27.01.2005 N 40) [Instructions for the identification, localization and elimination of source of quarantine weeds, approved by order of the Ministry of Agrarian Policy of Ukraine (27.01.2005 N 40)]. [cit. 2019-06-14].https://zakon.rada.gov.ua/laws/ show/z0201-05

Ipatov, V.S., Kirikova, L.A., Gerasimenko, G.G., 1996. Initial succession in the pine forests on sand. Bulletin of St. Petersburg State University, 17 (3): 26-30.

IUSS WORKING GROUP WRB, 2015. World reference base for soil resources 2014, update 2015: international soil classification system for naming soils and creating legends for soil maps. World Soil Resources Reports, No. 106. Rome: FAO. 203 p.

Kirilov, I., BANOv, M., 2017. Ecological characteristics of reclaimed areas in Pernik mines region, Bulgaria. Agricultural Science and Technology. 9 (2): 151-159. https:// doi.org/10.15547/ast.2017.02.027

KorzH, A.P., 2013a. Zhizneobespechennost populyatsiy kak osnova formirovaniya emkosti sredyi ih obitaniya [Survival of populations as the basis for the formation of the capacity of their habitat]. Science and Education a New Dimension: Natural and Technical Sciences, 15 (2): 31-
37.

KorzH, A.P., 2013b. Emkost sredyi: razvitie ponyatiya i ego soderzhanie [Capacity of the environment: the development of the concept and its content]. Ecology and Noospherology, 24 (1-2): 94-101.

Korzh, A.P., Akimov, I.A., Zahovalko, T.V., 2016. Emkost sredyi kak predposyilka dlya upravleniya sostoyaniem populyatsiy [The capacity of the environment as a prerequisite for managing the state of populations]. Biological Bulletin of Bogdan Chmelnitskiy Melitopol State Pedagogical University, 6 (3): 132-140.

Lovelock, J.E., 2000. Gaia a new look at life on earth. Oxford: University Press. 252 p.

Matveev, N.M., 2011. Osnovy stepnogo lesovedeniya professora A.L. Belgarda i ih sovremennaya interpretatsiya [Fundamentals of Prof. A.L. Belgard's steppe forestry conception and their modern interpretation]. Samara: Samara University Press. 126 p.

Montagu, K.D., Conroy, J.P., Atwell, B.J., 2001. The position of localized soil compaction determines root and subsequent shoot growth responses. Journal of Experimental Botany, 52: 2127-2133. http://dx.doi.org/10.1093/jex$\operatorname{bot} / 52.364 .2127$

Odling-Smee, F.J., Laland, K.N., Feldman, M.W., 2003. Comments on niche construction: the neglected process in evolution. Princeton: Princeton University Press. 468 p.

Raunkier Ch., 1937. Plant life forms. Oxford: Clarendon Press. 104 p.

Semenova-Tyan-Shanskaya, A.M., 1954. Biologiya rasteniy i dinamika rastitelnosti melovyih obnazheniy po r. Derkul [Plant biology and dynamics of vegetation in cretaceous outcrops along the Derkul river]. In Proceedings of the Botanical Institute of V. L. Komarov, Academy of Sciences of the USSR. Series III (Geobotany), vol. 9. Moscow, Leningrad: Publishing house of the USSR Academy of Sciences, p. 578-645.

Sheoran, V., Sheoran, A. S., Poonia, P., 2010. Soil reclamation of abandoned mine land by revegetation: a review. International Journal of Soil, Sediment and Water, 3 (2): article 13. [cit. 2019-05-12]. https://scholarworks.umass.edu/intljssw/vol3/iss2/13

Serafim, M.E., Vitorino, A.C.T., Рeixoto, P.P.P., Souza, C.M.A. Carvalho D.F., 2008. Intervalo hidrico otimo em um latossolo vermelho distroferrico sob diferentes sistemas de producao [Least limiting water range in a distroferric red latossol under different growing systems]. Engenharia Agricola, 28 (4): 654-665. http://dx.doi. org/10.1590/S0100-69162008000400005

SLuginova, I.S., 2009. Osobennosti floryi melovyih obnazheniy basseyna r. Polnoy (Rostovskaya obl.) [Features of floracretaceous outcrops in Polnaya river basin (Rostov region)]. Electronic Journal "Investigated in Russia”. 68: 904-915. [2019-04-16]. http://zhurnal.ape. relarn.ru/articles/2009/068.pdf.

StePanovskih, A.S., 2001. Ekologiya [Ecology]. Yuniti-Dana. $703 \mathrm{p}$.

Tarasov, V.V., 2012. Flora Dnipropetrovskoi i Zaporizkoi oblastei [Flora of the Dnipropetrovsk and Zaporizhia re- 
gions]. Lira: DNU. 276 p.

TOKHTAR, V.K., 2013. Research of formation features of the main ecological- and cenotypical groups in local flora of permanent establishment on study of landscape systems in the southwest of central Russian upland. Industrial Botany, 13: 43-49.

Ullah, M., Wolkenhauer, O., 2011. Stochastic approaches for systems biology. New York, Dordrecht, Heidelberg, London: Springer. $290 \mathrm{p}$.

Vanags, C., Minasny, B., McBratney, A.B., 2004. The dynamic penetrometer for assessment of soil mechanical resistance. In Supersoil 2004. Proceedings of the 3th Australian New Zealand Conference. University of Sydney, Australia, 5-9 December 2004. Gosford, N.S.W.: Regional Institute Ltd. [cit. 2019-04-21].

http://www.regional.org.au/au/asssi/supersoil2004/s14/ poster/1565_vanagsc.htm.

Vernadskiy, V.I., 1978. Zhivoe veschestvo [Living matter]. Moskva. $258 \mathrm{p}$.

Voron, E.A., 2010. Svoystva sozdavaemoy pochvyi pri posloynoy gornotehnicheskoy i biologicheskoy rekultivatsii [Properties of soil created by means of layerwise minetechnical and biological recultivation]. Scientific Bulletin of National Mining University, 5: 23-28.

YeterevsKa, L.V., Stammerer, G.F., Kanash A.P., 2008. Rekultyvovani grunty pidkhody do klasyfikatsii i systematyky [Recultivated soils approaches to classification and taxonomy]. Gruntiznavstvo, 9 (3-4): 147-150.

Zadorozhna, G.O., 2018. Spatiotemporal dynamics of soil penetration resistance of recultivated soil. Ekológia (Bratislava), 37: (3): 82-89.

Zadorozhna, G.O., Andrusevych, K.V., Zhukov, O.V., 2018. Soil heterogeneity after recultivation: ecological aspect. Folia Oecologica, 45 (1): 46-52. doi: https://doi. org/10.2478/foecol-2018-0005

Zavialova, L.V., 2017. Vydy invazyvnyh roslyn, njebjezpjechni dlja pryrodnogo fitoriznomanittja ob'ektiv prirodno-zapovidnogo fondu Ukrayni [The most harmful invasive plant species for native phytodiversity of protected areas of Ukraine]. Biological Systems, 19 (1): 87-107.

Zhuкоv, O.V., 2009. Ekomorfichniy analiz konsortsiy gruntovykh tvaryn [Ecomorph analysis of soil animal consortium]. Dnipropetrovsk: Svidler A.L. 239 p.

Zhukov, A.V., Zadorozhnaya, G.A., Andrusevich, K.V., 2012. Ekolohicheskiie kharakteristiki pedonov diernovolitohiennykh pochv na lesakh [Ecological characteristics of the pedons of soddy-lithogenic soils on loess]. Vistnyk Djerzhavnogo Dnipropetrovs'kogo Agrarnogo Universytetu, 2: 9-11.

Zhukov, A.V., Zadorozhnaya, G.A., 2015. Otsenka ecomorphogenesa pedozema y chernozema obyknovennoho na osnove pokazatelei tverdosty [Pedozem and chernozem ecomorphogenesis assessment by soil penetration resistance data]. Ahrokhimiya i Gruntoznavstvo, 84: 72-80.

Zhukov, A., Zadorozhnaya, G., 2016. Spatial heterogeneity of mechanical impedance of atypical chernozem: the ecological aproach. Ekológia (Bratislava), 35 (3): 263-278.

Zhukov, A.V., ZadorozhnaYA, G.A., 2017. The dynamics of the replantozems spatial heterogeneity. Principles of the Ecology, 1 (12): 226-237.

Received June 25, 2019 Accepted November 11, 2019 